\title{
Exploratory Analysis of the Airspace Throughput and Sensitivities of an Urban Air Mobility System
}

\author{
Kenneth $\mathrm{H}$ Goodrich ${ }^{1}$ and Bryan E. Barmore ${ }^{2}$ \\ NASA Langley Research Center, Hampton VA, 23681
}

\begin{abstract}
The use of small, vertical-takeoff and landing aircraft to provide efficient, high-speed, ondemand passenger transportation within a metropolitan area (e.g. intra-city transportation) is a topic of increasing interest and investment within the aerospace and transportation communities. Preliminary, mostly vehicle-level analysis suggests that passenger-carrying "Urban Air Mobility" has the potential to provide meaningful door-to-door trip time savings compared to identical trips taken solely by automobile, even for relatively short trips of a few tens of miles. Subsequent analysis has shown that if such trips can be conducted at costs competitive with ground transportation, the demand for such flight operations, not surprisingly, becomes unprecedented by historical airspace operations counts, raising fundamental questions regarding feasibility, practicality, capacity and basic system attributes such as separation criteria. In this paper, we conduct a preliminary assessment of vertipad requirements and en route separation minima relative to the feasibility of large-scale urban aviation operations. This analysis is acknowledged as being far from comprehensive and is intended to help define the initial boundaries of an airspace system compatible with enabling high-volume operations.
\end{abstract}

\section{Introduction}

$\mathrm{W}$ ith the rapid advance of technologies that could enable safe, easy-to-use, cost-effective, on-demand transportation using small vertical-takeoff and landing (VTOL) aircraft, several groups have proposed passenger-carrying, "Urban Air Mobility" (UAM) capabilities intended to augment existing transportation options for intra-city travel, including operations to/from neighboring suburbs while avoiding potentially congested roads [1,2]. While the term UAM encompasses carriage of both persons and cargo, in this paper, we focus on carriage of passengers in aircraft that can carry on the order of four or fewer passengers. Recent media reports document that a wide range of companies are developing such aircraft, with several currently flying prototypes [3]. Many of publically available assessments of this concept to date have focused on the operations of individual vehicles such as done in reference 1 or predictions of total, travel demand created by individual vehicle attributes (e.g. trip speed and cost) if they can be realized, en masse such as done in reference [4]. Relatively little analysis or even discussion has centered around the feasibility and requirements of an airspace system that can facilitate sufficient UAM operations for it to result in a transportation system with enough capacity to be available, to "meaningful" numbers of travelers as they move around a metropolitan area. While the term meaningful is without a hard definition in this paper, it is intended to suggest shifting $\sim 5 \%$ or more of long-distance but still intra-metropolitan area trips (e.g. $\sim 20-100$ miles) from roads to the air. For major metropolitan areas this equates to tens to hundreds of thousands of passenger trips per day and might involve hundreds to eventually thousands of aircraft simultaneously operating over a metro area. It should be noted that while it is recognized that aircraft operations at such levels raise significant additional concerns such as community safety and noise that must be addressed to enable broad, public acceptance, these topics are beyond the scope of this brief paper.

To develop better insight into how UAM airspace operations might differ from current national airspace system (NAS) operations, let us first briefly consider the current air and ground transportation systems. Air transportation as experienced by most of the traveling public today is a form of mass-transportation with high-occupancy aircraft carrying large numbers of travelers between a relative small numbers of origin-destination pairs. According to the

\footnotetext{
${ }^{1}$ Senior Researcher, Dynamics Systems and Control Branch, k.goodrich@nasa.gov, Senior AIAA Member

${ }^{2}$ Acting Deputy Project Manager for Technology for Air Traffic Management -Exploration project, Crew Systems and Aviation Operations Branch, bryan.barmore@nasa.gov, AIAA Member.
} 
Bureau of Transportation Statistics [5], in 2015 the US commercial air carrier aircraft fleet consisted of approximately 7,000 aircraft which traveled 7.8 billion vehicle miles travel (VMT) while providing 641 billion passenger mile of service (i.e. average occupancy of 82 passengers per aircraft). These aircraft provided connectivity between 531 US airports with scheduled commercial air service, with $94 \%$ of this traffic going through the busiest 100 airports. In contrast, travel by light passenger vehicle (i.e. cars and light trucks) is typically highly distributed and personalized with individual travelers or groups choosing the origin, destination, and departure time of trips. In 2015 the lightpassenger vehicles in the US consisted of approximately 243 million registered vehicles, which accumulated 3.1 trillion VMT and 3.8 trillion passenger miles (i.e. average occupancy of 1.2 persons per vehicle), while traveling between hundreds of millions of origin-destination pairs (i.e. street address pairs in this case). Perhaps the most striking aspect of this comparison is the difference in vehicle occupancy levels between commercial air and ground transportation (i.e. a factor of 68) and resulting large differences in the number of vehicle operations and VMT required for to accommodate a given number of passenger miles. Given that aircraft operations today require miles and/or minutes of separation vs a few seconds and feet for cars, it seems likely that the feasibility of UAM depends in large part on an airspace system that can safely and efficiently support unprecedented numbers of vehicle operations in all phases of flight and in close proximity with minimal delay or disruptions.

Given the relatively large temporal separation that might be expected based on current practices and operations between safe, successive operations at typical aircraft takeoff and landing areas (vertipads for VTOL aircraft), UAM system capacity is likely to initially be constrained by vertipad availability (i.e. number of available vertipads) and the maximum operational throughout of each vertipad (i.e. the maximum number of vehicle operations accommodated by a vertipad in hour) In many areas, such as urban centers, the high density of potential travelers (i.e. high, localized trip demand) combined with limited suitable vertipad siting options is likely to make such vertipad-based bottlenecks a persistent challenge to UAM system capacity, regardless of other factors. However, many other areas within a typical metroplex have a surprisingly large number of potential vertipad sites [1] that could be developed over time, easing ground-side bottlenecks and increasing the possibility that airborne conflicts become an increasingly important factor in system scalability and efficiency. In this paper, we use relatively simple techniques to explore and develop more refined engineering insight into of the fundamental roles that ground infrastructure and in-flight separation requirements may play in the capacity and efficiency of an evolving UAM transportation system. The expectation is that this background will help inform airspace system research and development for integration of UAM in to the NAS

\section{Vertipad Analysis}

In this section, we consider estimates of the potential aircraft and passenger throughput of individual vertipads where a vertipad can support a single VTOL operation at a time. We put this throughput into the context of an airspace system by estimating the number of vertipads needed to support predicted passenger travel through a network of vertiports that are part of a UAM transportation system. A vertiport is taken to be 1 or more vertipads in close proximity that function as an integrated arrival/departure node within the UAM system.

Since developing an optimized, geographically situated and corresponding demand predications for a hypothesized transportation system with speculative service characteristics (e.g. speed, wait-time, reservation requirements, coverage, comfort, etc.) and price is obviously a needed but specialized and speculative undertaking, in the following analysis, rather than develop our own concept, we leverage concepts and projections Uber's Elevate project presented in public forums to stimulate discussion $[2,6,7]$. Because these concepts and projections are drawn from several sources, separated by some months in time, they should not be taken to reflect any specific plans by Uber.

Starting with the description of a 25 -vertiport network for the Dallas-Fort Worth area as presented by German and McDonald at the 2017 Uber Elevate Summit [6] and shown in figure 1, we combined this description with total trip demand estimates shown in figure 2 for "early-scaled" operations as discussed by Moore in [7] and as shown in keynote presentation at the 2017 Rotorcraft Business and Technology Summit in Ft. Worth Texas. The combination of these two figures allows estimation of predicated travel through each vertiport which is needed to estimate the vertipad requirements. We focused on the "early, scaled operations" (ESO) phase for two reasons in this analysis. First, ESO is expected better consistency between the several Uber sources as this phase is more likely to be tied to demand projections of sufficient confidence to justify initial investments. Second, from the perspective of needed vertipad capacity, the ESO phase starts operations at what are expected to be the highest demand locations (e.g. 

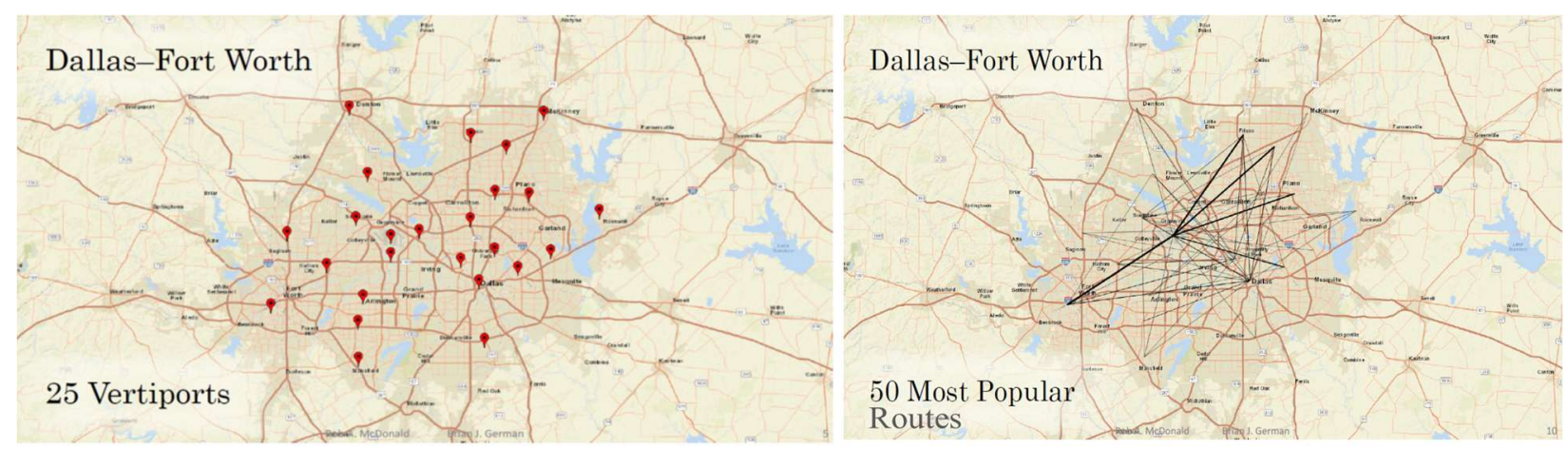

\section{Uber Supplied Demand Model}
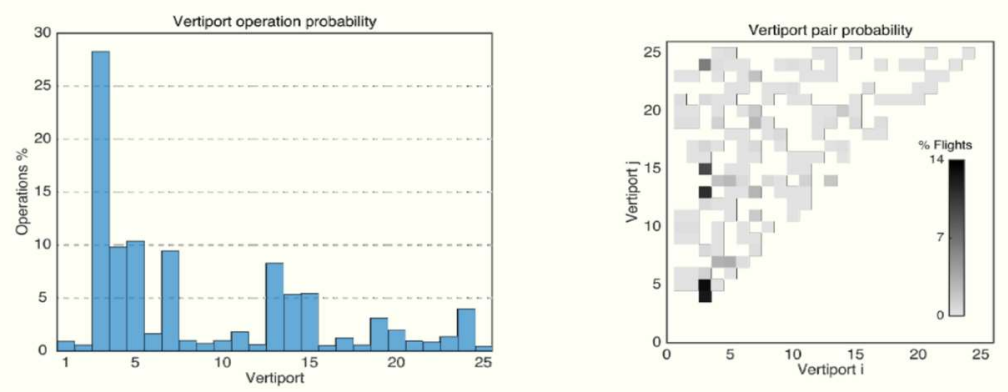

Most flights involve DFW airport.

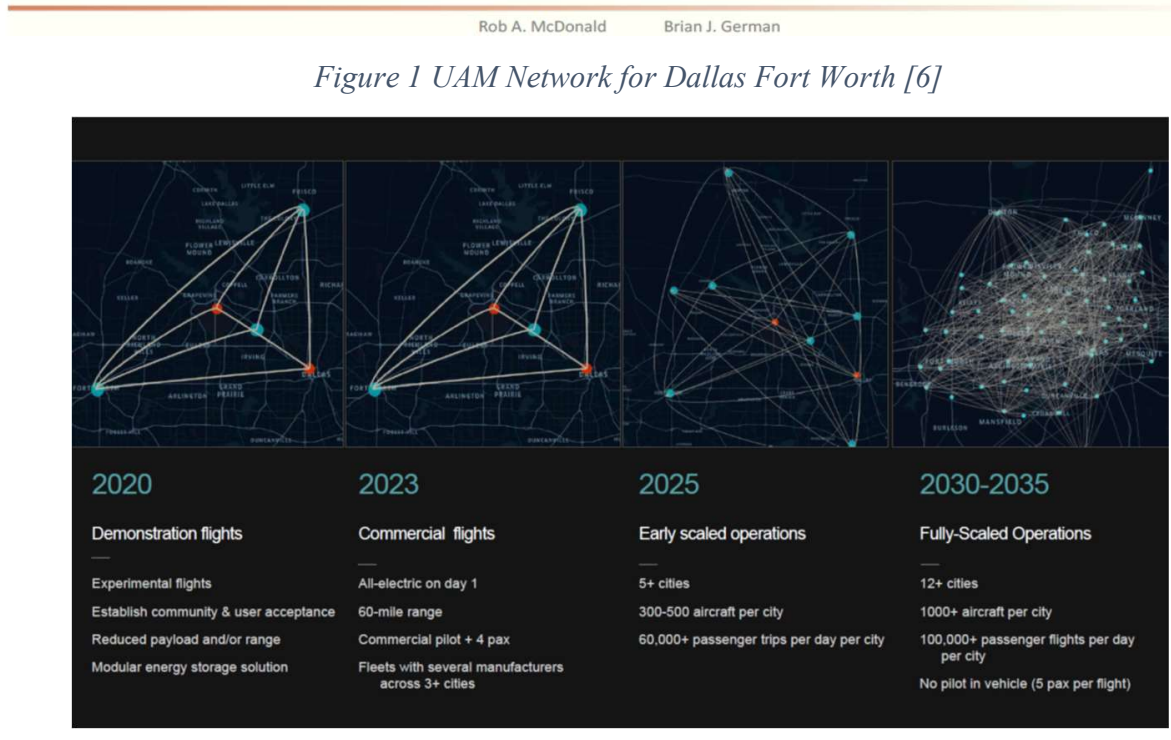

Figure 2 Total Demand Prediction [7]

commercial airports, central downtown locations, and key outlaying suburban spokes) and these are likely to remain the very high demand locations even as the system scales. As shown in figure 1, the seven most utilized vertiports capture an estimated $75 \%$ of the total demand. Additional vertipads tend to make the system more distributed and convenient, but generate relatively small demand increments.

By combining the two Elevate sources, we use simple spreadsheet analysis to estimate the peak, off-peak, and average hourly operational demands for the individual vertiports in the network. Peak and off-peak demand estimates are based on 16 hour days with half of the demand occurring in 2, 3 hour morning and evening "peaks" and the other half of the demand occurring in the remaining, 10 off-peak hours. Based on the Elevate white paper [2], aircraft were 
assumed to have 4 passenger seats and to operate with an average load factor of $67 \%$ (i.e. 2.68 passengers per aircraft). Two operations per aircraft (i.e. takeoff and landing) are required for one flight. In practice, given the potential need for "deadhead" legs to reposition aircraft from where they are to where there needed, for example during a peak period with a net flow into a city center, the ratio of operations to passenger trips may be worse than modeled in this analysis where deadhead legs are not considered. The results of this analysis are shown in tables 1 and 2, first for the entire network (table 1) and then for the individual vertiports (table 2). It is interesting to note that vertiport \#3, which serves the Dallas Ft. Worth (DFW) Airport has by far, the highest demand with 16,800 daily passenger trips. This number is approximately $13 \%$ of the 125,000 daily trips made to DFW using various ground transportation modes [8].

\begin{tabular}{|c|c|c|c|}
\hline Time Period & $\begin{array}{c}\text { Passenger } \\
\text { Trips }\end{array}$ & $\begin{array}{c}\text { Aircraft } \\
\text { Flights }\end{array}$ & $\begin{array}{c}\text { Number of } \\
\text { Operations }\end{array}$ \\
\hline Day & 60000 & 22388 & 44776 \\
\hline Hour, peak & 5000 & 1866 & 3731 \\
\hline Hour, average & 3750 & 1399 & 2799 \\
\hline Hour, off-peak & 3000 & 1119 & 2239 \\
\hline
\end{tabular}

Table 1 Total Hourly Demand Estimates

\begin{tabular}{|c|c|c|c|c|c|c|c|}
\hline $\begin{array}{l}\text { Vertiport } \\
\text { Index }\end{array}$ & $\begin{array}{l}\text { Percentage of } \\
\text { Operations }\end{array}$ & $\begin{array}{c}\text { Travelers Per } \\
\text { Hour, Peak }\end{array}$ & $\begin{array}{l}\text { Travelers } \\
\text { Per Hour, } \\
\text { Average } \\
\end{array}$ & $\begin{array}{c}\text { Travelers } \\
\text { Per Hour, } \\
\text { Off-Peak } \\
\end{array}$ & $\begin{array}{c}\text { Operations } \\
\text { Per Hour, } \\
\text { Peak } \\
\end{array}$ & $\begin{array}{c}\text { Operations } \\
\text { Per Hour, } \\
\text { Average } \\
\end{array}$ & $\begin{array}{c}\text { Operations } \\
\text { Per Hour, } \\
\text { Off-Peak } \\
\end{array}$ \\
\hline 1 & $1.0 \%$ & 50.0 & 37.5 & 30.0 & 37.3 & 28.0 & 22.4 \\
\hline 2 & $0.5 \%$ & 25.0 & 18.8 & 15.0 & 18.7 & 14.0 & 11.2 \\
\hline 3 & $28.0 \%$ & 1400.0 & 1050.0 & 840.0 & 1044.8 & 783.6 & 626.9 \\
\hline 4 & $10.0 \%$ & 500.0 & 375.0 & 300.0 & 373.1 & 279.9 & 223.9 \\
\hline 5 & $10.0 \%$ & 500.0 & 375.0 & 300.0 & 373.1 & 279.9 & 223.9 \\
\hline 6 & $1.5 \%$ & 75.0 & 56.3 & 45.0 & 56.0 & 42.0 & 33.6 \\
\hline 7 & $9.5 \%$ & 475.0 & 356.3 & 285.0 & 354.5 & 265.9 & 212.7 \\
\hline 8 & $1.0 \%$ & 50.0 & 37.5 & 30.0 & 37.3 & 28.0 & 22.4 \\
\hline 9 & $1.0 \%$ & 50.0 & 37.5 & 30.0 & 37.3 & 28.0 & 22.4 \\
\hline 10 & $1.0 \%$ & 50.0 & 37.5 & 30.0 & 37.3 & 28.0 & 22.4 \\
\hline 11 & $2.0 \%$ & 100.0 & 75.0 & 60.0 & 74.6 & 56.0 & 44.8 \\
\hline 12 & $1.0 \%$ & 50.0 & 37.5 & 30.0 & 37.3 & 28.0 & 22.4 \\
\hline 13 & $8.0 \%$ & 400.0 & 300.0 & 240.0 & 298.5 & 223.9 & 179.1 \\
\hline 14 & $5.0 \%$ & 250.0 & 187.5 & 150.0 & 186.6 & 139.9 & 111.9 \\
\hline 15 & $5.0 \%$ & 250.0 & 187.5 & 150.0 & 186.6 & 139.9 & 111.9 \\
\hline 16 & $0.5 \%$ & 25.0 & 18.8 & 15.0 & 18.7 & 14.0 & 11.2 \\
\hline 17 & $1.0 \%$ & 50.0 & 37.5 & 30.0 & 37.3 & 28.0 & 22.4 \\
\hline 18 & $1.0 \%$ & 50.0 & 37.5 & 30.0 & 37.3 & 28.0 & 22.4 \\
\hline 19 & $3.0 \%$ & 150.0 & 112.5 & 90.0 & 111.9 & 84.0 & 67.2 \\
\hline 20 & $2.5 \%$ & 125.0 & 93.8 & 75.0 & 93.3 & 70.0 & 56.0 \\
\hline 21 & $1.0 \%$ & 50.0 & 37.5 & 30.0 & 37.3 & 28.0 & 22.4 \\
\hline 22 & $1.0 \%$ & 50.0 & 37.5 & 30.0 & 37.3 & 28.0 & 22.4 \\
\hline 23 & $1.0 \%$ & 50.0 & 37.5 & 30.0 & 37.3 & 28.0 & 22.4 \\
\hline 24 & $4.0 \%$ & 200.0 & 150.0 & 120.0 & 149.3 & 111.9 & 89.6 \\
\hline 25 & $0.5 \%$ & 25.0 & 18.8 & 15.0 & 18.7 & 14.0 & 11.2 \\
\hline Totals & $100.0 \%$ & 5000 & 3750 & 3000 & 3731 & 2799 & 2239 \\
\hline
\end{tabular}

The next step is estimating the number vertipads needed accommodate the predicated operations at each vertiport. This step requires an estimate of the expected, peak operations rate a vertipad can accommodate combined with a lower, scheduled utilization rate that results in an average delay time acceptable to the overall operation. If the actual utilization rate is greater than or equal to the maximum throughput (e.g. demand for actual operations due to passenger arrivals, either by ground or flight, occur at a rate equal to or exceeding a vertipads maximum operational rate), basic 
queueing theory highlights that the queue to use the pad will grow to infinity. For the current analysis, we somewhat arbitrarily assume that an average queueing time up to 4 minutes is acceptable. For an on-demand system, the length of the queue could also be managed in real-time via dynamic, congestion-pricing, but in practice this would tend to make the cost/benefit of the service less predictable and discourage it's use.

Considering first, the expected maximum operational rate of a vertipad, relatively little real-world data is available do to the scarcity of advanced, UAM aircraft. Helicopter data may be useful, but due to limited demand, these operations today generally are not conducted as sustained, high-tempo operations. There are isolated examples of specialized, high-capacity civilian helicopter operations such used to occur on race day at British Grand Prix in near Silverstone England. In 1999, it is reported that $\sim 4200$ helicopter operations ( 2100 trips) took place in one day between dawn and dusk, suggesting an average rate of 262 operations per hour [9]. However, multiple helipads were used at this event and the number and throughput of individual pads is not known to the authors. Military helicopter operations also achieve high operational tempos but this is usually associated with pre-coordinated and highly structured formation operations that are probably not compatible with highly distributed UAM flight operations. For UAM, the Elevate white paper [2] assumes 60 seconds per takeoff and 75 seconds per landing for an average operational of 67.5 seconds per operation and 53 operations per hour per vertipad. Syed, et al [4] use estimates of arrival and departure lengths and speeds and arrive at an estimate of 45 operations per hour. For the purposes of this paper, a theoretical maximum of 50 operations per hour (takeoff or landing) is used in the analysis. It is worth noting that currently, twin-engine helicopters operating in densely populated areas with limited availability of emergency landing sites are often required to use take-off and land using "category A" procedures [10]. The gist these procedures is to assure that in the event of an engine failure at any time, the helicopter is either positioned to directly return to the pad from which it is operating from or has sufficient altitude to safely transition to forward flight with a positive rate of climb. These procedures usual involve near vertical ascent or descent segments in proximity to the pad and result in lower pad operational rates then used in this paper. The applicability of category-A-like procedures for UAM operations from vertipads located in constrained, densely populated areas needs to be assessed in more detail as it could significant affect vertipad and vertiport productivity

Using a basic $\mathrm{M} / \mathrm{M} / 1$ queueing model [11] combined with the target average wait time that is under 4 minutes suggests that a vertipad with a theoretical maximum of 50 operations per hour should be scheduled for a no more than 38 operations per hour. This utilization ratio of 0.76 provides a buffer needed to recover from likely operational variations such as a departing aircraft occupying the pad longer the usual due to a passenger concern or an temporary influx of travelers. Using the rate of 38 operations per hour to convert the demanded operations shown in table 2 to the required number of vertipads for the 25 vertiports results in table 3 . While the vertipad count to satisfy peak demand is of primary interest since these typically would determine infrastructure requirements, counts matched to average and off-peak demand are also shown.

By coincidence, the vertipad count for the peak scenario works out to approximately 1 vertipad per percent of total demand moving through a given vertiport. Since most vertipads only capture a few percent of the total demands, 1 or 2 vertipads are needed at these locations and may be operational feasible with limited development of suitable procedures. It should be recognized that the pads are assumed to be able to conduct simultaneous, independent operations which requires sufficient separation between the two final approach and takeoff areas for FATO (currently $>200$ ' for helicopters) and separate arrival and departure paths (and corresponding obstacle-free approach/departure protection surfaces) for each FATO. Several of the proposed vertiports, in particular \#3, require a very large number of vertipads, particularly when considering that they would ideally conduct simultaneous operations, independent from each another and from other air traffic associated with DFW and the several other significant airports in the area. Strategies for reducing the needed vertiport count at these high-demand locations include operating aircraft with high load-factors, ideally filling all passenger seats; using larger aircraft with more seats; developing higher-throughput and capacity procedures; and as a last-resort, managing demand via pricing. It is likely that vertiports with more than 2 vertipads will require procedures supporting simultaneous, dependent operations (i.e. operations will be highly coordinated). This dependence will create potential couplings of delay between the multiple pads. Space permitting, very large vertiports such as $3 \#$, are likely to benefit in terms of tempo and increased independence of operations by being subdivided and spatially distributed into smaller, vertiports.

In the following section, we consider the safe, efficient movement of aircraft once airborne. 


\begin{tabular}{|c|c|c|c|c|c|c|c|}
\hline $\begin{array}{l}\text { Vertiport } \\
\text { Index }\end{array}$ & $\begin{array}{c}\text { Percentage of } \\
\text { Operations }\end{array}$ & $\begin{array}{c}\text { Travelers } \\
\text { Per Hour, } \\
\text { Peak } \\
\end{array}$ & $\begin{array}{l}\text { Travelers } \\
\text { Per Hour, } \\
\text { Average }\end{array}$ & $\begin{array}{l}\text { Travelers } \\
\text { Per Hour, } \\
\text { Off-Peak } \\
\end{array}$ & $\begin{array}{c}\text { Vertipad } \\
\text { Count, } \\
\text { Peak } \\
\end{array}$ & $\begin{array}{c}\text { Vertipad } \\
\text { Count, } \\
\text { Average } \\
\end{array}$ & $\begin{array}{c}\text { Vertipad } \\
\text { Count, } \\
\text { Off-Peak }\end{array}$ \\
\hline 1 & $1.0 \%$ & 50.0 & 37.5 & 30.0 & 1.0 & 0.7 & 0.6 \\
\hline 2 & $0.5 \%$ & 25.0 & 18.8 & 15.0 & 0.5 & 0.4 & 0.3 \\
\hline 3 & $28.0 \%$ & 1400.0 & 1050.0 & 840.0 & 27.5 & 20.6 & 16.5 \\
\hline 4 & $10.0 \%$ & 500.0 & 375.0 & 300.0 & 9.8 & 7.4 & 5.9 \\
\hline 5 & $10.0 \%$ & 500.0 & 375.0 & 300.0 & 9.8 & 7.4 & 5.9 \\
\hline 6 & $1.5 \%$ & 75.0 & 56.3 & 45.0 & 1.5 & 1.1 & 0.9 \\
\hline 7 & $9.5 \%$ & 475.0 & 356.3 & 285.0 & 9.3 & 7.0 & 5.6 \\
\hline 8 & $1.0 \%$ & 50.0 & 37.5 & 30.0 & 1.0 & 0.7 & 0.6 \\
\hline 9 & $1.0 \%$ & 50.0 & 37.5 & 30.0 & 1.0 & 0.7 & 0.6 \\
\hline 10 & $1.0 \%$ & 50.0 & 37.5 & 30.0 & 1.0 & 0.7 & 0.6 \\
\hline 11 & $2.0 \%$ & 100.0 & 75.0 & 60.0 & 2.0 & 1.5 & 1.2 \\
\hline 12 & $1.0 \%$ & 50.0 & 37.5 & 30.0 & 1.0 & 0.7 & 0.6 \\
\hline 13 & $8.0 \%$ & 400.0 & 300.0 & 240.0 & 7.9 & 5.9 & 4.7 \\
\hline 14 & $5.0 \%$ & 250.0 & 187.5 & 150.0 & 4.9 & 3.7 & 2.9 \\
\hline 15 & $5.0 \%$ & 250.0 & 187.5 & 150.0 & 4.9 & 3.7 & 2.9 \\
\hline 16 & $0.5 \%$ & 25.0 & 18.8 & 15.0 & 0.5 & 0.4 & 0.3 \\
\hline 17 & $1.0 \%$ & 50.0 & 37.5 & 30.0 & 1.0 & 0.7 & 0.6 \\
\hline 18 & $1.0 \%$ & 50.0 & 37.5 & 30.0 & 1.0 & 0.7 & 0.6 \\
\hline 19 & $3.0 \%$ & 150.0 & 112.5 & 90.0 & 2.9 & 2.2 & 1.8 \\
\hline 20 & $2.5 \%$ & 125.0 & 93.8 & 75.0 & 2.5 & 1.8 & 1.5 \\
\hline 21 & $1.0 \%$ & 50.0 & 37.5 & 30.0 & 1.0 & 0.7 & 0.6 \\
\hline 22 & $1.0 \%$ & 50.0 & 37.5 & 30.0 & 1.0 & 0.7 & 0.6 \\
\hline 23 & $1.0 \%$ & 50.0 & 37.5 & 30.0 & 1.0 & 0.7 & 0.6 \\
\hline 24 & $4.0 \%$ & 200.0 & 150.0 & 120.0 & 3.9 & 2.9 & 2.4 \\
\hline 25 & $0.5 \%$ & 25.0 & 18.8 & 15.0 & 0.5 & 0.4 & 0.3 \\
\hline Totals & $100.0 \%$ & 5000 & 3750 & 3000 & 98 & 74 & 59 \\
\hline
\end{tabular}

Table 3 Vertipad Estimates

\section{Airborne Analysis}

Another potential limiter to UAM operations is safe and efficient movement through the airspace. Ensuring vehicles do not collide or encounter wake vortices from other vehicles is a major component of ensuring safe movement of air traffic. Under instrument flight rules, several layers of collision and wake vortex protection are provided from sequencing and spacing aircraft into and out of dense areas such as airports or weather-constricted airspace, to the provision of separation assurance, to on-board collision avoidance systems. Depending on the specific conditions, IFR separation requirements generally range from three to five nautical miles laterally and $1000-2000 \mathrm{ft}$ vertically. Under visual flight rules, collision avoidance is provided by the pilot with "see and avoid" techniques with no stated ranges. However, recent work for Unmanned Aerial Systems have defined acceptable detect-and-avoid (DAA) definitions for use when a pilot is not visually scanning for other traffic. RTCA DO-365 [12] defines this DAA area as a horizontal range (DMOD/HMD*) of $4000 \mathrm{ft}$ and a time component of $\tau_{\text {mod }}=35 \mathrm{sec}$. Unlike RADAR separation requirements, the Well Clear definition combines a fixed lateral distance with a time-based parameter. This applies to vehicles operating in Class D, E, and G airspace excluding operations in the VRF traffic pattern of an airport. On-going work is looking at the appropriate values for low-speed, low-altitude operations such as the traffic pattern at an airport.

UAM operations are going to need to operate in instrument meteorological conditions at densities well beyond current IFR capabilities. Mueller et al [13] identified the ineffectiveness of see-and-avoid capabilities, especially in IMC, as a critical barrier to general UAM operations. This section will provide a first-order assessment of how close together vehicles need to get to each other to enable the density and tempo of operations envisioned for UAM. Further work on how safe operations will be implemented for UAM operations and if they will fall under existing FAA rules or new rules will need to be developed. A full safety analysis is needed to set any separation or collision avoidance areas. This work is meant to be a first pass to determine the likely range of values to be considered and assess the difficulty of the problem. If future UAM densities can be supported with existing DAA values, then it is expected that 
the desired safety levels will be able to be met. However, if significantly smaller values are needed, then new technologies or techniques will be needed to ensure safe operations at the desired demand.

Our approach was to take a set of flights and determine the number of encounters experienced that would have required maneuvering to maintain a safe distance from other traffic. For simplicity, we will refer to these encounters as losses of separation although final safe distance requirements may be in terms of well clear or a yet-to-be-defined quantity. Looking at metrics such as the number of encounters and the time spent in a loss of separation event, will give a rough assessment of what that safe separation value will need to be.

Traffic demand model based on by Syed, et al [3] was used to generate the simulated flights. One thousand vertiports were placed in the Northern California/Bay Area based on US census data. Flight demand was based on a variety of cost and time requirements. For the purpose of the current study, this data was used to generate 40,000 flights over a three hour period. To model likely vehicle performance, flights were assigned a cruise altitude from $1000 \mathrm{ft}$ to $5000 \mathrm{ft}$ in $500 \mathrm{ft}$ increments based on flight distance. No consideration was used for terrain avoidance. For simplification, a vertical separation of $400 \mathrm{ft}$ was required so each cruise level was separated from all others. This gives a set of ten independent altitude layers. It is assumed that the vehicles do not change altitude during their cruise phase of flight. A simple climb and descent model was used for arrivals and departures. While accurate vehicle dynamics will be necessary for operationally detecting and avoiding these encounters, they should not materially affect this analysis. Likewise, no environmental conditions, such as winds, were models and no additional sources of surveillance or behavior uncertainty were introduced. A simple departure scheduler was used to ensure that a flight was not generate already in a loss of separation with a vehicle departing from the same or near-by vertiport. No scheduling was used for arrival traffic as that was out of scope for this analysis. Managing arrival delay involves forecasting demand and propagating actions backwards along the intended flight. While another critical service needed for an operational system, it was out of scope for this initial assessment.

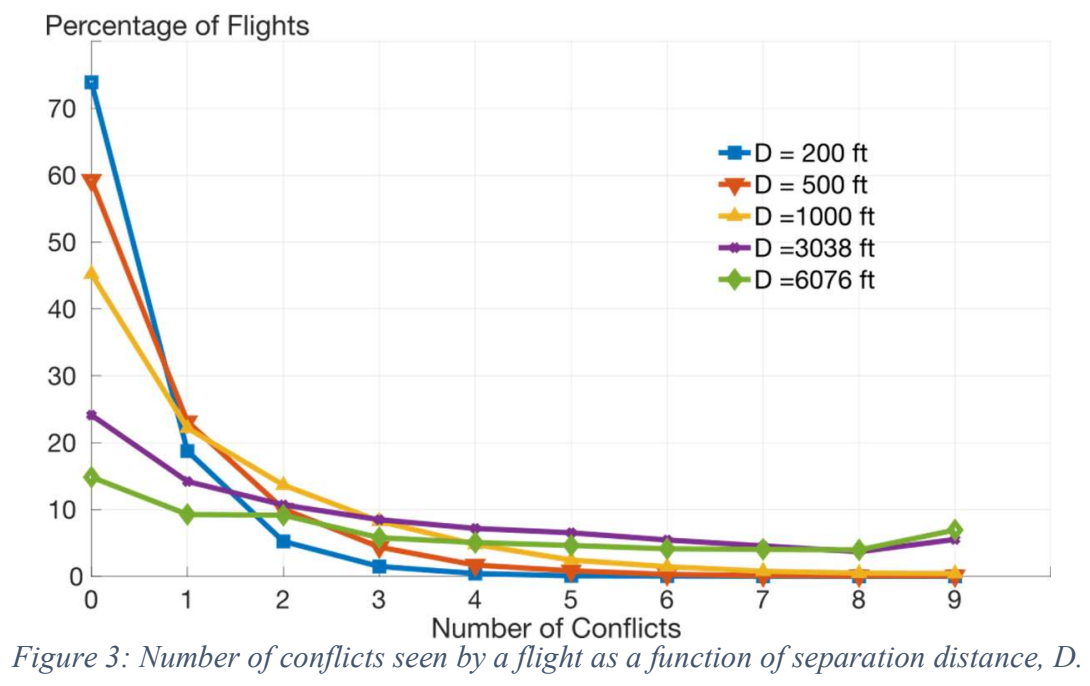

During the simulation runs, data was collected on when each loss of separation started and stopped, as well as how many losses of separation each vehicle was experiencing. Lateral separation values between $200 \mathrm{ft}$ and one nautical mile (6076 ft) where tested. As expected, increasing the separation value increased the number of conflicts. There was a nearly linear relationship $\left(R^{2}=0.998\right)$. Figure 3 shows percentage of flights experiencing different numbers of conflicts. Each line represents a different separation distance. At $200 \mathrm{ft}$ separation, over $90 \%$ of flights experienced zero or one conflict during their flight. At $6076 \mathrm{ft}$ separation, only $24 \%$ of flights had zero or one conflict with a median of 6 conflicts per flight. Similarly, at the larger separation distances, the flights spend more of their flight time in a loss of separation than at the smaller separation distances.

Figure 4 shows the median and inter-quartile range for the number of conflicts experienced per flight. The blue box represents the central $50 \%$ of the counts with the whiskers extended $1.5 \mathrm{x}$ the inter-quartile distance. The red line represents the median value. The red crosses are values that are outside the whisker. Note that since the count is necessarily an integer, many data points may appear as one cross. The median value for $200 \mathrm{ft}$ and $500 \mathrm{ft}$ separation is zero. 


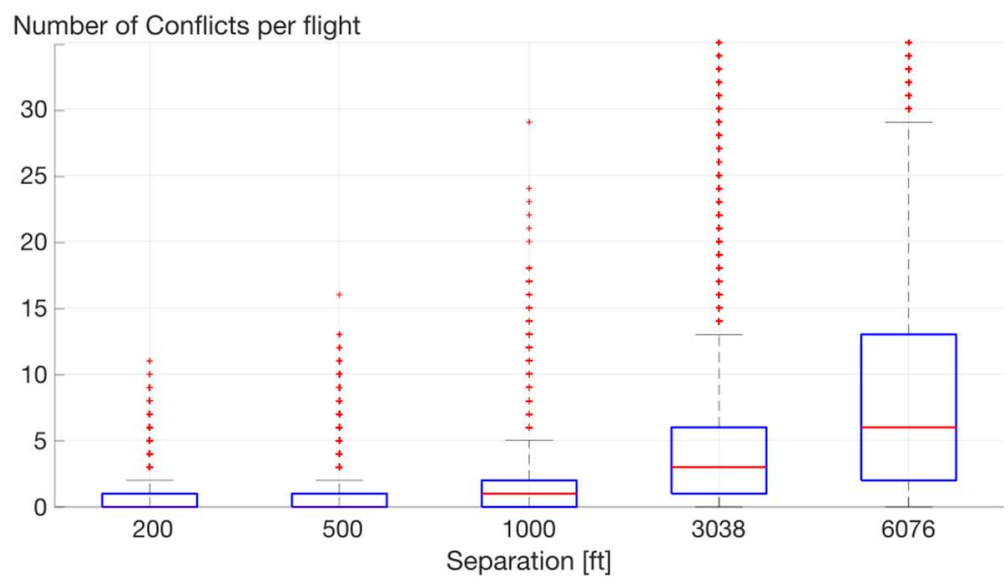

Figure 4: Box-and-whisker plot of number of losses of separation experienced per flight.

Additional runs looked at using the current well clear formula with horizontal separations, HMOD, for $500 \mathrm{ft}$ and $1000 \mathrm{ft}$ horizontal distance and $\tau_{-} \bmod =15 \mathrm{sec}$. The overall behavior is unchanged but the number of loss of separation events increased. For the HMOD $=500 \mathrm{ft}$ case, the likelihood of encountering a loss of separation as shown in Figure 3 , approached the $\mathrm{D}=1000 \mathrm{ft}$ case. This increase in the number of losses of separation encountered is expected since the circular values correspond to tau $=0$ for the effect of the well clear definition is to add additional protected space along the relative velocity vector.

A case study of a flights that encountered several conflicts shows that many of these occur as the flight was approaching the destination or leaving its origin, including interactions with flights going to nearby vertiports. Figure 5 shows one such case. The flight of reference is highlighted with a circle and a heavier black line, and is located in the upper right corner. The flight paths of all encounters are shown in blue dashed lines. The majority of losses of separation occur near the destination where there are several nearby vertiports and no arrival scheduling. A second case study showed similar behavior but the encounters were clustered around the origin vertiport and represented uncoordinated departures and arrivals from neighboring vertiports.

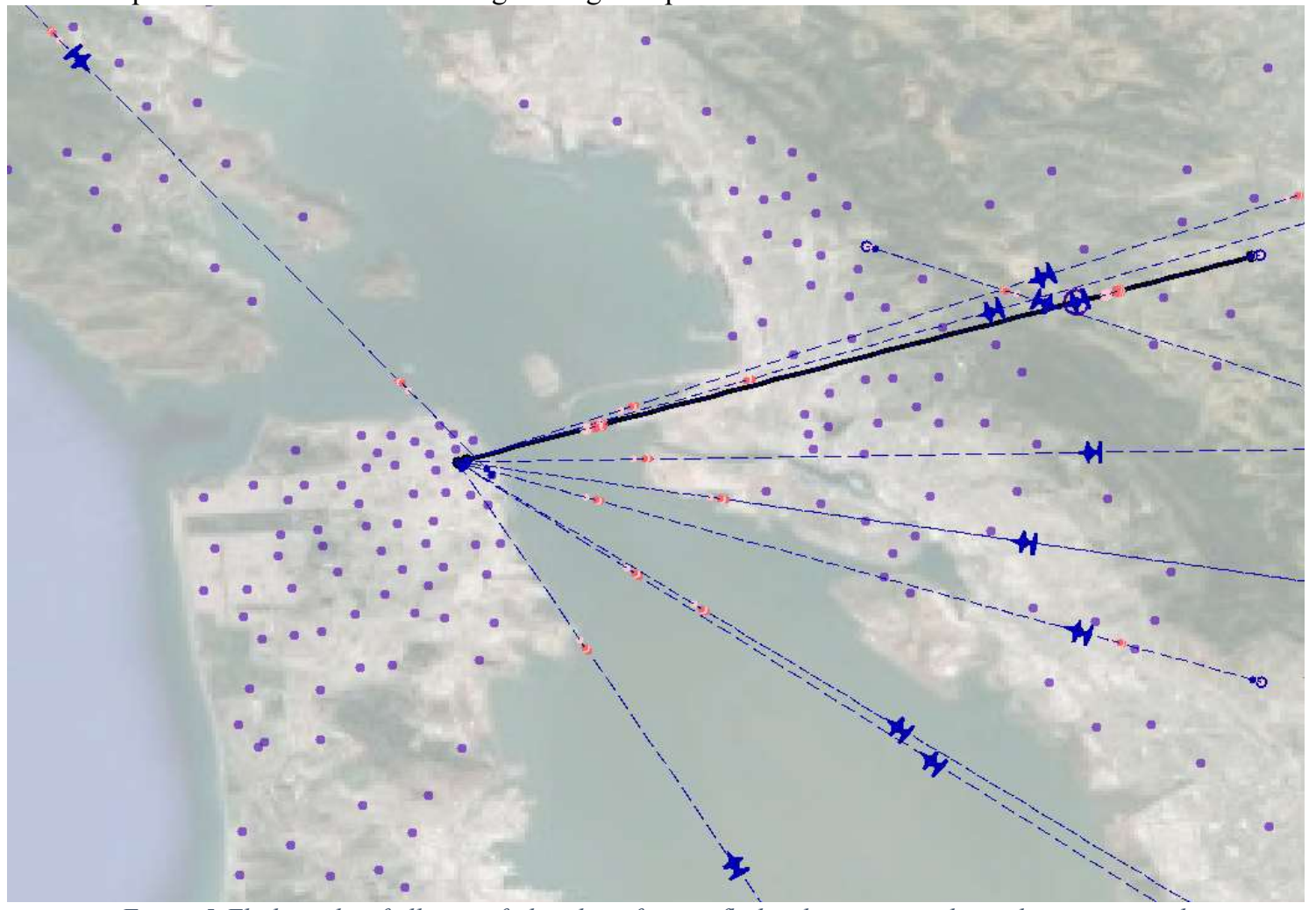

Figure 5:Flight paths of all aircraft that the reference flight, shown in purple circle, encountered. 


\section{Summary}

In this paper, our objective was to develop a better understanding of the airspace system demands of passengercarrying UAM. We approached this objective by using UAM travel demand estimates from several early studies and estimating the resulting traffic through vertiports and vertipads along with assessing time-referenced trajectories and the frequency of conflicts as a function of minimum separation standards.

The results of the vertipad analysis suggest that many areas of a metropolitan area can be served by relatively conventional ground infrastructure and operations, at least in terms of vehicle throughput rates modest numbers of vertipads in close proximity. That said, there are also likely to be key nodes that attract very high traffic levels and require a density of ground infrastructure and operations that is well beyond our current experience base. These nodes will be particularly interesting and important to assess and enable in more detailed studies of airspace concepts and operations.

The separation study was a first pass at identifying the range of values that future separation requirements would need to take to support high-demand, high-tempo UAM operations. This is just the first step in the long process of defining requirements to keep UAM vehicles safely separated from other UAM vehicles and non-UAM vehicles. It is clear that arrival and departure management will be critical and will likely involve multiple vertiports interacting. By setting operationally acceptable numbers of interactions, this work will provide a starting point for future, in-depth analysis. For example, if a median of one encounter to resolve per flights extending for no more than $20 \%$ of the flight time, was acceptable, the circular horizontal distance would likely be in the range of $1000 \mathrm{ft}-3000 \mathrm{ft}$.

In addition to vertipad and spacing considerations, there are a host of other issues that must be considered for the safe, successful integration of UAM operations into the NAS and our communities. Like synergistic efforts to integrate unmanned aircraft systems (UAS) into the NAS, UAM integration is likely to be a long-term undertaking that will require the airspace community to more deeply understand and assess foundational assumptions and implementations of today's system. Ultimately, the capacity, efficiency, productivity and safety the entire NAS a likely to benefit from these efforts.

\section{References}

[1] Antcliff, K. R., Moore, M. D., and Goodrich, K. H., "Silicon Valley as an Early Adopter for On-Demand Civil VTOL Opertations," In AIAA Aviation Technology, Integration, and Operations Conference, AIAA, 2016. doi: 10.2514/6.20163466

[2] Holden, J., and Goel, N., Fast-Forwarding to a Future of On-Demand Urban Air Transportation, San Francisco: 2016.

[3] Wakabayashi, D. "Flying Taxis May Be Years Away, but the Groundwork is Accelerating," The New York Times, p. B1, 1 March 2018.

[4] Syed, N., Rye, M., Ade, M., Trani, A., Hinze, N., Swingle, H., Smith, J., Marien, T., and Dollyhigh S., "Preliminary Considerations for ODM Air Traffic Management based on Analysis of Commuter Passenger Demand and Travel Patterns for the Silicon Valley Region of California," In 17th AIAA Aviation Technology, Integration, and Operations Conference, AIAA 2017-3082, June 2017

[5] https://www.bts.gov/topics/national-transportation-statistics

[6] McDonald, R., and German B., "eVTOL Stored Energy Overview”, Uber Elevate Summit, 2017, https://www.uber.com/info/elevate/summit2017/

[7] Sanchez, N., "Space Cowboys: Dallas-Fort Worth to be First Area Worldwide to Test UberAir Flying Cars in 2020," Fort Worth Business Press, 26 September 2017

[8] Nelson, Nygard Consulting Associates Inc. "Dalls/Fort Worth International Airport Transit Service Planning Study, Final Report," November 2012

[9] Drwiega, A., “A Day at the (Motor) Races Beats the Recession”, Rotor \& Wing International, 29 May, 2012, http://www.rotorandwing.com/2012/05/29/a-day-at-the-motor-races-beats-the-recession/

[10] McConKey, E., Hawley, R., Anoll, R., "Helicopter Rejected Takeoff Airspace Requirements,” DOT/FAA/RD-90/7, August 1991

[11] Gross, D., Shortle, J., Thompson, J., Harris, C., Fundamentals of Queuing Theory, $4^{\text {th }}$ Edition, Wiley and Sons Inc., ISBN13: 978-0471791270, 2008

[12] "Minimum Operational Performance Standards (MOPS) for Detect and Avoid (DAA) Systems," RTCA, DO-365, Washington, D.C., May 2017.

[13] Mueller, E., Kopardekar, P., and Goodrich, K., "Enabling Airspace Integration for High-Density Mobility Operations," 17th AIAA Aviation Technology, Integration, and Operations Conference, AIAA AVIATION Forum, AIAA 2017-3086, Jun. 2017. 